\title{
Paramedics Have Untapped Potential to Address Social Determinants of Health in Canada
}

\section{Potentiel inexploité des ambulanciers paramédicaux pour la question des déterminants sociaux de la santé au Canada}

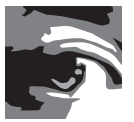

AMIR ALLANA, PCP, BASC

MSc Candidate, Health Services Research

Institute of Health Policy, Management and Evaluation

Dalla Lana School of Public Health

University of Toronto Fellow

McNally Project for Paramedicine Research

Graduate Student

Upstream Lab, MAP/Centre for Urban Health Solutions

Li Ka Shing Knowledge Institute

Unity Health Toronto

Toronto, ON

ANDREW D. PINTO, MD, CCFP, FRCPC, MSC

Associate Professor

Institute of Health Policy, Management and Evaluation

Dalla Lana School of Public Health

University of Toronto

Scientist and Director

Upstream Lab, MAP/Centre for Urban Health Solutions

Li Ka Shing Knowledge Institute

Unity Health Toronto

Clinician Scientist

Department of Family and Community Medicine

Faculty of Medicine

University of Toronto

Staff Physician

Department of Family and Community Medicine

St. Michael's Hospital

Toronto, ON 


\begin{abstract}
The role of paramedics, including select paramedics providing primary and preventive care in homes and community settings, is evolving in health systems around the world. These developments are associated with improvements in health outcomes, improved access to services and reduced emergency department use. Building on these existing trends in paramedicine, and because social conditions contribute to illness and are strong predictors of future health service use, addressing patients' social needs should be integrated into core paramedic practice in Canada. We discuss how paramedic education, culture and governance could better enable paramedics to address the social determinants of health.
\end{abstract}

\title{
Résumé
}

Le rôle des ambulanciers paramédicaux, notamment ceux qui fournissent des soins primaires et préventifs à domicile ou en milieu communautaire, est en pleine évolution dans les systèmes de santé du monde entier. Ces changements sont associés à une amélioration des résultats cliniques, à un meilleur accès aux services et à une utilisation réduite des services d'urgence. Dans la foulée des tendances actuelles en matière de services paramédicaux - et puisque les conditions sociales influent sur l'état de santé et constituent de forts prédicteurs de l'utilisation des services de santé - il faudrait intégrer les besoins sociaux des patients dans la pratique paramédicale de base au Canada. Nous discutons de la façon dont l'éducation, la culture et la gouvernance paramédicales pourraient mieux permettre aux ambulanciparamédicaux de tenir compte des déterminants sociaux de la santé.

\section{Introduction}

Social determinants of health are "the conditions of daily life, the circumstances in which people are born, grow, live, work and age" and include our income, housing conditions, employment status, food security and social support networks (World Health Organization 2008). Epidemiological studies have strongly supported the influence of social determinants on morbidity, mortality and health status. Precise estimates vary, but it is likely that at least half of the differences in health status observed between groups can be explained by social factors (Keon and Pépin 2009). Social determinants have been found to predict who becomes a frequent user of health services, including paramedic services and emergency departments (Fitzpatrick et al. 2015).

Paramedics regularly engage with patients who have complex health and social service needs, including people with precarious housing and employment status and those with chronic addiction and mental health conditions (McCann et al. 2018). Not all emergency calls are acute, and up to $50 \%$ of cases that paramedics attend may not need care at an emergency room (Snooks et al. 2013). As mobile healthcare professionals who spend one-on-one time with patients, paramedics are uniquely positioned to understand and address the social factors that contribute to patients' health. 
In this commentary, we explore how paramedics can address the social determinants of health. We draw on literature from around the world and discuss the implications for Canadian jurisdictions.

\section{Paramedicine Is Evolving}

Paramedics have historically only been responsible for providing medical care before and during transport to an emergency department (ED). This is changing: paramedicine has evolved over the last two decades, in both acute and non-acute settings. Specially trained paramedics in many jurisdictions conduct medical, social and environmental assessments and provide preventative care to patients without transport to a hospital. In other cases, they transport patients to alternative destinations or refer them directly to follow-up services. This is known as community paramedicine, also sometimes referred to as mobile integrated healthcare in the US (Bigham et al. 2013). Originally conceived to extend access to services for rural, underserved and vulnerable populations, community paramedicine programs now exist in many countries, including Canada, the UK, the US and Australia (Choi et al. 2016). In the UK, Australia and New Zealand, there are also extended care paramedics and paramedic practitioners providing out-of-hospital care. The breadth and scope of programs vary, exist in both urban and rural settings and are context-specific. A systematic review found that community paramedicine is associated with improved health outcomes, reductions in healthcare spending and reduced ED use (Bigham et al. 2013). These programs also tend to increase patient satisfaction (Dainty et al. 2018) and are examples of patient-centred, integrated care (Rasku et al. 2019).

These newer models of paramedic care suggest that paramedics are capable of addressing social factors when appropriately educated and supported. In Canada, most community paramedicine programs are targeted at a small number of patients and are not available to everyone. Over $70 \%$ of these programs operate through scheduled home visits, where patients have been selected using operationally driven criteria such as being a "frequent caller" or at-risk for hospital readmission (Chan et al. 2019). Less than 20\% of programs include initiatives that occur on an unscheduled emergency call. As such, assessing and treating for social determinants is not integrated into front-line paramedics' scope of practice, but rather, it is left to a small number of community paramedics treating a relatively small number of patients.

\section{Integrating a Social Determinants Lens into Core Paramedic Practice}

Not all paramedics can or should be community paramedics, and not all patients need the in-depth case management that community paramedicine typically offers (Leyenaar et al. 2018). However, given that many emergency calls are non-urgent, exacerbated by social factors (Agarwal et al. 2019) and related to gaps in primary care (Booker et al. 2014), all paramedics could play a role in addressing social determinants. Two specific ways in which 
core paramedic practice could be enhanced are (i) conducting better social and environmental assessments and (ii) directly conferring with primary care teams and community-based organizations.

\section{Conducting social and environmental assessments}

Several projects and robust trials have evaluated the impact of paramedics conducting social assessments. In Hamilton, Ontario, community paramedics utilize lifestyle-based risk questionnaires and pre-specified algorithms to refer patients to preventative care (Agarwal et al. 2018). Another study validated paramedic use of a clinical decision tool to assess risk factors for independence loss in the elderly and initiate preventative care (Lee et al. 2016). Most municipalities in Ontario have integrated some form of Community Referral by Emergency Medical Services, a program whereby any paramedic on an emergency call can initiate community paramedicine follow-up on the basis of factors such as risk of falls, medication non-compliance, poor hygiene and caregiver burnout (MOHLTC 2017). Similar programs exist in Manitoba and Alberta.

Paramedics on emergency calls could be further equipped with tools and questionnaires to assess patients for risks associated with housing, income and food security. Studies from primary care and pediatrics suggest that these social needs can be addressed. A growing number of clinically validated tools are available for the assessment of these risks. Paramedic services could make such tools available to their staff, educate them on social assessment and encourage them to gather the patients' social history where relevant. When paramedics interact with patients in the community, they can document important contextual and circumstantial information about a patient's living conditions that impact their health. This must be done with explicit patient consent and in a manner that is sensitive to privacy concerns. Current patient-care records could be updated for fields to contain this information. As electronic medical records become more integrated, these assessments could be shared, with patient consent, with other members of the care team to assist with care planning and activation of additional services.

\section{Directly conferring with primary care teams and community-based organizations}

Thorough assessments can help paramedics identify unmet social and health service needs and gaps in care. While in some cases it may be appropriate to refer a patient to community paramedicine, paramedics on an emergency call could also consider directly contacting other members of the patient's care team, including family physicians and social workers. This would imply a shift in the norms of paramedicine toward shared responsibility for care continuity, rather than simply transporting a patient to an ED where patients may not receive that level of care coordination (Hjälte et al. 2007).

Paramedics could be oriented to the social services and agencies that operate in their 
area. These include legal aid, housing, food banks, shelters, detox centres and employment agencies. Paramedic services could establish agreements that allow staff to confer with these agencies while on an emergency call and directly refer patients based on agreed-upon criteria. This would allow paramedics to expand the range of options they can exercise to address the social determinants of health. While nearly $40 \%$ of community paramedicine programs already collaborate with community services such as detox facilities and mental health hospitals (Chan et al. 2019), these options are unavailable to most Canadian paramedics on emergency calls.

\section{Implications for Education, Culture and Governance}

To better enable paramedics to address social determinants of health, change is needed in at least three broad, interconnected areas: education; culture; and governance and payment models. Healthcare is under provincial jurisdiction in Canada and some paramedic services are under municipal control. While there are common themes, any changes would need to be adapted to uniquely local contexts across the country.

\section{Education}

Currently, all Canadian paramedics are educated at the diploma or certificate level through vocational institutes and private colleges. Their curriculums are governed by standards set by provincial licensing bodies or Ministries of Education and informed by the National Occupational Competency Profile for paramedics (NOCPs). These competencies focus on knowledge and skills in emergency medicine. Assessing social risks, integration with community services and patient advocacy are not emphasized. Only the small number of paramedics who become community paramedics, often later in their career, receive supplemental training in these topics. The NOCPs could be updated to include knowledge and skills about social determinants and primary care coordination. Recent work on developing a Canadian paramedic profile (Tavares et al. 2016) is a promising step, and it could help inform a new framework for paramedic education in Canada. This would further be aided by establishing faculties or departments of paramedicine at universities with investment in curriculum development and pedagogical research.

Paramedic curricula worldwide are undergoing reform to reflect the changing role of paramedics in health systems (Hou et al. 2013), with the UK, Australia and New Zealand establishing bachelors- and masters-level degree programs in paramedicine (O'Meara et al. 2017). This transformation is starting in Canada as well. Ontario is moving toward threeyear advanced diplomas as a minimum entry to practice for paramedics. A few universities now offer degrees in paramedicine, including in Alberta, Ontario and Prince Edward Island; however, degrees are not mandatory for practice in Canada. Transition to university settings presents an opportunity for paramedic educators to collaborate with faculties of nursing, social work and medicine. Paramedics need a theoretical foundation in topics such as how social factors impact health, power and privilege and the role of race, gender and 
socio-economic striations in contributing to health inequity. This could be supplemented by teaching paramedics how to elicit a social history during patient care, collaborate with different providers in the community and consider how inequities impact patients' experiences in the health system. Paramedic programs could also consider partnering with social service organizations and local clinics to expose paramedic students to underserved populations through field placements and coach them on how to interact with a diversity of patients. These changes would pave the way for a generation of paramedics better equipped to address social determinants.

\section{Culture of paramedicine}

Paramedicine in many western nations, including Canada, has its roots in trauma and transport medicine practised by returning soldiers after the World Wars (Shah 2006). Despite the complex social problems such as mental health, poverty and substance abuse that paramedics frequently encounter, there are conflicting views within the profession on what is within their scope of practice (McCann et al. 2018). Paramedics are still taught and indoctrinated with the sentiment that a paramedic's role is to respond to high-acuity biological emergencies and rapidly transport patients to a hospital. The organizational cultures and operational realities they operate within reinforce these ideas, and paramedics performing roles such as referrals to community services can experience "role confusion" (Brydges et al. 2015).

There is need for the paramedic profession in Canada to update its definition of a "paramedic" and embrace an identity that more fully reflects the broad spectrum of primary and emergency care paramedics now provide. This will help normalize practices such as assessing for social determinants and conferring with other care organizations. National associations such as the Paramedic Chiefs of Canada and the Paramedic Association of Canada as well as provincial and municipal paramedic labour unions all need to agree on a unified paramedic identity. This needs to be reflected in their branding and messaging to help the paramedic workforce coalesce around shared principles and enable other health providers to better collaborate with paramedics.

\section{Governance and payment models}

Paramedic governance varies significantly across Canada. Historically, paramedic scope of practice has been defined by a small number of emergency physicians with a narrow focus on treating acute emergencies such as trauma and cardiac arrest. However, there is a global trend toward professionalization and self-regulation (Maguire et al. 2016). Five Canadian provinces now have self-governing colleges of paramedicine. There have been similar developments in the UK, Australia and New Zealand over the past two decades. As paramedics in Canada become independent clinicians leading their own profession, regulatory bodies could support practices to address the social determinants of health. These topics could be integrated into mandatory continuing medical education and included as formal clinical guidelines for paramedics. 
Paramedic services tend to be funded on the basis of the number of patients transported and evaluated on the speed of their response time. As such, paramedic organizations are not necessarily incentivized to address social determinants of health especially when they do not involve transport to a hospital (Munjal et al. 2019). Changing this requires legislative or regulatory reform by provincial governments, as it relates to healthcare billing and performance indicators. The form these changes take depends on the organizational relationship between paramedics and the health system, which varies between the provinces. In provinces such as British Columbia (BC), paramedicine is a centralized provincial service managed by the Provincial Health Services Authority. This is in contrast with Saskatchewan, Ontario and Quebec, where hundreds of municipal and private organizations provide paramedic services. BC, Alberta and Nova Scotia now have "treat and release" and "treat and refer" guidelines, whereby paramedics can provide some alternate services to transport, but how these costs are accounted for is unclear. The Ontario government is in the early stages of piloting payment mechanisms for what they call "new models of care" provided by paramedics on emergency calls (Government of Ontario 2019). Better integration with the health system or new payment models for paramedic services may help realign the incentive to address social determinants, particularly where there are cost savings that occur in other parts of the health system as a result of paramedic care.

\section{Conclusion}

As complex needs and aging populations strain healthcare systems across Canada, we need to find creative and innovative ways to utilize existing resources. Paramedics are part of these untapped resources, which, if utilized wisely, can help reduce the healthcare burden from social determinants and improve quality of care. This is compatible with existing trends in paramedic education, culture and governance. As provinces across the country re-think the role of paramedics in their health systems, the time is ripe to integrate social determinants of health into core paramedic practice.

\section{Acknowledgements and Support}

The authors appreciate the helpful remarks on previous versions of this manuscript by Dr. Gina Agarwal and Dr. Kerry Kuluski.

Correspondence may be directed to: Amir Allana, Institute for Health Policy, Management and Evaluation, University of Toronto, 155 College Street, 4th floor, Toronto, ON M5T 3M6. He can be reached by e-mail ata.allana@utoronto.ca.

\section{References}

Agarwal, G., R. Angeles, M. Pirrie, B. McLeod, F. Marzanek, J. Parascandalo et al. 2018. Evaluation of a Community Paramedicine Health Promotion and Lifestyle Risk Assessment Program for Older Adults Who Live in Social Housing: A Cluster Randomized Trial. CMAJ 190(21): E638-47. doi:10.1503/cmaj.170740. 
Agarwal, G., J. Lee, B. McLeod, S. Mahmuda, M. Howard, K. Cockrell et al. 2019. Social Factors in Frequent Callers: A Description of Isolation, Poverty and Quality of Life in Those Calling Emergency Medical Services Frequently. BMC Public Health 19(1): 684. doi:10.1186/s12889-019-6964-1.

Bigham, B.L., S.M. Kennedy, I. Drennan and L.J. Morrison. 2013. Expanding Paramedic Scope of Practice in the Community: A Systematic Review of the Literature. Prehospital Emergency Care 17(3): 361-72. doi:10.3109/ 10903127.2013.792890.

Booker, M.J., R.L. Simmonds and S. Purdy. 2014. Patients Who Call Emergency Ambulances for Primary Care Problems: A Qualitative Study of the Decision-Making Process. Emergency Medicine Journal 31(6): $448-$ 52. doi:10.1136/emermed-2012-202124.

Brydges, M., C. Spearen, A. Birze and W. Tavares. 2015. A Culture in Transition: Paramedic Experiences with Community Referral Programs. Canadian Journal of Emergency Medicine 17(6): 631-38. doi:10.1017/ cem.2015.6.

Chan, J., L.E. Griffith, A.P. Costa, M.S. Leyenaar and G. Agarwal. 2019. Community Paramedicine: A Systematic Review of Program Descriptions and Training. Canadian Journal of Emergency Medicine 21(6): 749-61. doi:10.1017/cem.2019.14.

Choi, B.Y., C. Blumberg and K. Williams. 2016. Mobile Integrated Health Care and Community Paramedicine: An Emerging Emergency Medical Services Concept. Annals of Emergency Medicine 67(3): 36166. doi:10.1016/j.annemergmed.2015.06.005.

Dainty, K.N., M.B. Seaton, I.R. Drennan and L.J. Morrison. 2018. Home Visit-Based Community Paramedicine and Its Potential Role in Improving Patient-Centered Primary Care: A Grounded Theory Study and Framework. Health Services Research 53(5): 3455-70. doi:10.1111/1475-6773.12855.

Fitzpatrick, T., L.C. Rosella, A. Calzavara, J. Petch, A.D. Pinto, H. Manson et al. 2015. Looking Beyond Income and Education: Socioeconomic Status Gradients among Future High-Cost Users of Health Care. American Journal of Preventive Medicine 49(2): 161-71. doi:10.1016/j.amepre.2015.02.018.

Government of Ontario. 2019, September 5. Enabling New Models of Care for Select 9-1-1 Patients. Retrieved June 28, 2020. <https://www.ontariocanada.com/registry/view.do?postingId=30268\&language=en $>$.

Hjälte, L., B.O. Suserud, J. Herlitz and I. Karlberg. 2007. Why Are People Without Medical Needs Transported by Ambulance? A Study of Indications for Pre-Hospital Care. European Journal of Emergency Medicine 14(3): 151-56. doi:10.1097/MEJ.0b013e3280146508.

Hou, X., J. Rego and M. Service. 2013. Review Article: Paramedic Education Opportunities and Challenges in Australia. Emergency Medicine Australasia 25(2): 114-19. doi:10.1111/1742-6723.12034.

Keon, W.J. and L. Pépin. 2009, June. A Healthy, Productive Canada: A Determinant of Health Approach. The Standing Senate Committee on Social Affairs, Science and Technology Final Report of Senate Subcommittee on Population Health. Retrieved November 14, 2018. <https://sencanada.ca/content/sen/Committee/402/popu/ rep/rephealth1jun09-e.pdf>.

Lee, J.S., R.P. Verbeek, M.J. Schull, L. Calder, I.G. Stiell, J. Trickett et al. 2016. Paramedics Assessing Elders at Risk for Independence Loss (PERIL): Derivation, Reliability and Comparative Effectiveness of a Clinical Prediction Rule. Canadian Journal of Emergency Medicine 18(2): 121-32. doi:10.1017/cem.2016.14.

Leyenaar, M., B. McLeod, J. Chan, W. Tavares, A. Costa and G. Agarwal. 2018. A Scoping Study and Qualitative Assessment of Care Planning and Case Management in Community Paramedicine. Irish Journal of Paramedicine, 3(1): 1-15. doi:10.32378/ijp.v3i1.76.

Maguire, B., P. O'Meara and A. Newton. 2016. Toward an International Paramedic Research Agenda. Irish Journal of Paramedicine 1(2): 1-3. doi:10.32378/ijp.v1i2.38.

McCann, T.V., M. Savic, N. Ferguson, E. Bosley, K. Smith, L. Roberts et al. 2018. Paramedics' Perceptions of Their Scope of Practice in Caring for Patients with Non-Medical Emergency-Related Mental Health and/or Alcohol and Other Drug Problems: A Qualitative Study. PLoS ONE 13(12): e0208391. doi:10.1371/journal. pone. 0208391.

Ministry of Health and Long-Term Care (MOHLTC). 2017, July. Community Paramedicine Framework for Planning, Implementation and Evaluation. Retrieved November 28 2018. <https://www.renfrewparamedics.ca/ wp-content/uploads/2017/07/CP-Framework-2017_07_18.pdf>. 
Paramedics Have Untapped Potential to Address Social Determinants of Health in Canada

Munjal, K.G., G.S. Margolis and A.L. Kellermann. 2019. Realignment of EMS Reimbursement Policy: New Hope for Patient-Centered Out-of-Hospital Care. JAMA 322(4): 303-04. doi:10.1001/jama.2019.7488.

O'Meara, P.F., S. Furness and R. Gleeson. 2017. Educating Paramedics for the Future: A Holistic Approach. Health and Human Services Administration 40(2): 219-51.

Rasku, T., M. Kaunonen, E. Thyer, E. Paavilainen and K. Joronen. 2019. The Core Components of Community Paramedicine - Integrated Care in Primary Care Setting: A Scoping Review. Scandinavian Journal of Caring Sciences 33(3): 508-21. doi:10.1111/scs.12659.

Shah, M.N. 2006. The Formation of the Emergency Medical Services System. American Journal of Public Health 96(3): 414-23. doi:10.2105/AJPH.2004.048793.

Snooks, H.A., M.R. Kingston, R.E. Anthony and I.T. Russell. 2013. New Models of Emergency Prehospital Care That Avoid Unnecessary Conveyance to Emergency Department: Translation of Research Evidence into Practice? The Scientific World Journal 2013: 1821202. doi:10.1155/2013/182102.

Tavares, W., R. Bowles and B. Donelon. 2016. Informing a Canadian Paramedic Profile: Framing Concepts, Roles and Crosscutting Themes. BMC Health Services Research 16: 477. doi:10.1186/s12913-016-1739-1.

World Health Organization (WHO). 2008. Closing the Gap in a Generation: Health Equity through Action on the Social Determinants of Health - Final Report of the Commission on Social Determinants of Health. Retrieved November 14, 2018. <https://www.who.int/publications/i/item/WHO-IER-CSDH-08.1>.

\section{Longwoods Virtual Breakfast Series}

\section{Past meetings include:}

The 'Now Normal':

Transitions in Health

Services During and

Beyond the Pandemic

Featuring

Jennifer Zelmer

President and CEO,

Canadian Foundation for

Healthcare Improvement
The Lessons of COVID-19 for Canadian Learning Health Systems

Featuring

Rick Glazier

Scientific Director of the Institute of Health Services and Policy Research at the Canadian Institutes of Health Research

\section{The Changing Faces of Digital Leadership \\ Featuring \\ Lydia Lee \\ Partner, KPMG Canada}

\section{Duska Kennedy}

Chief Digital Officer,

North York General Hospital

F. Cigi Osler

Chair, Canadian Medical Forum

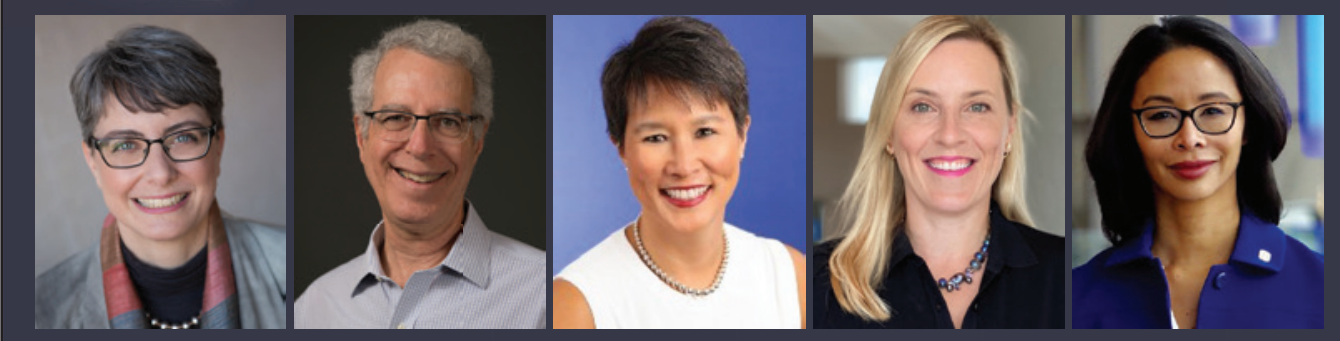

Sign up at longwoods.com/newsletters to be notified of dates and registration 